\title{
La datation des roches par la thermoluminescence. Applications
}

G. Valladas

\section{Citer ce document / Cite this document :}

Valladas G. La datation des roches par la thermoluminescence. Applications. In: Bulletin de l'Association française pour l'étude du quaternaire, vol. 16, n¹-2, 1979. pp. 43-52;

doi : https://doi.org/10.3406/quate.1979.1345

https://www.persee.fr/doc/quate_0004-5500_1979_num_16_1_1345

Fichier pdf généré le 06/11/2020 


\title{
LA DATATION DES ROCHES PAR LA THERMOLUMINESCENCE. APPLICATIONS
}

\author{
par G. VALLADAS*
}

\section{I. - INTRODUCTION}

\section{A. Le phénomène}

Certains minéraux qui ont été soumis au rayonnement de corps radioactifs émettent de la lumière quand on les chauffe. Si on les chauffe une $2^{\text {ème }}$ fois ils n'émettent plus de lumière. Si on les irradie à nouveau ils sont capables de reémettre de la lumière par chauffage. Ce phénomène appelé thermoluminescence est donc dû à l'effet des rayonnements.

De tels minéraux existent dans la nature. Ce sont la fluorine, le quartz, le feldspath... Ils sont continuellement irradiés par les radioéléments naturels présents dans toute roche : l'uranium, le thorium et le potassium. Les radio-éléments formant la famille de l'Uranium 238 et du Thorium 232 se désintégrent en émettant des particules chargés $\alpha$ et $\beta$ et des rayons $\gamma$. La désintégration du potassium 40 se fait par l'émission de particules $\beta$ et de rayons $\gamma$.

Ces rayonnements traversant le minéral ionisent les atomes. Certains des électrons libérés sont repris dans des défauts cristallins d'où ils ne peuvent sortir sans un apport d'énergie. Ces défauts sont nommés pièges. Comme le rayonnement agit sans cesse, le nombre d'électrons fixés sur les pièges croît avec le temps (ce processus est analogue au noircissement d'une plaque photographique qui augmente avec la duré d'exposition à la lumière). Lors d'une chauffe, les électrons sont libérés de leurs pièges et ils vont rejoindre les atomes qui ont perdu un électron. Au cours de cette réorganisation du cristal, ils peuvent exciter des centres lu- minogènes. La quantité de lưmière produite est d'autant plus grande que la quantité de rayonnement a été plus importante donc le temps d'irradiation plus long.

La grandeur qui exprime le mieux cette quantité de rayonnement est la densité d'énergie cédée au cristal par ce rayonnement (une partie de cette énergie est consommé dans le transfert des électrons sur les pièges). Cette densité d'énergie est nommée dose d'irradiation. Lorsqu'un rayonnement a perdu 100 ergs par gramme de matière traversée celle-ci a reçu 1 rad, par définition.

Le processus de thermoluminescence tel que nous le considérons dans une datation se résume donc ainsi :

- accumulation d'une excitation particulière dans le minéral par l'action des rayons $\alpha, \beta$ et $\gamma$.

- émission de lumière au cours d'une chauffe à $400-500^{\circ}$ du minéral. La quantité de lumière est proportionnelle à la dose totale de rayonnement reçue pendant le temps qui s'est écoule depuis la formation du minéral (une calcite, par exemple) ou le précédent réchauffement à une température suffisante pour que toute l'excitation antérieure ait été effacée (ainsi que le quartz d'un morceau de granite pris dans la lave). Si la concentration en radioéléments dans le minéral et dans sa roche n'a pas varié, la dose totale qu'il a reçue est proportionnelle au temps : dose totale $=$ dose annuelle $x$ âge.

La mesure de la thermoluminescence d'un mineral est la réunion de deux opérations (fig. 1):

- la chauffe : l'échantillon étant placé sur une plaque chauffante dans une enceinte obscure, on procède à une montée en température. Elle doit se faire en présence d'un gaz inerte afin d'éviter l'action de

\footnotetext{
* Centre des Faibles Radioactivités, Laboratoire mixte CNRS-CEA, 91190 Gif-sur-Yvette (France).
} 


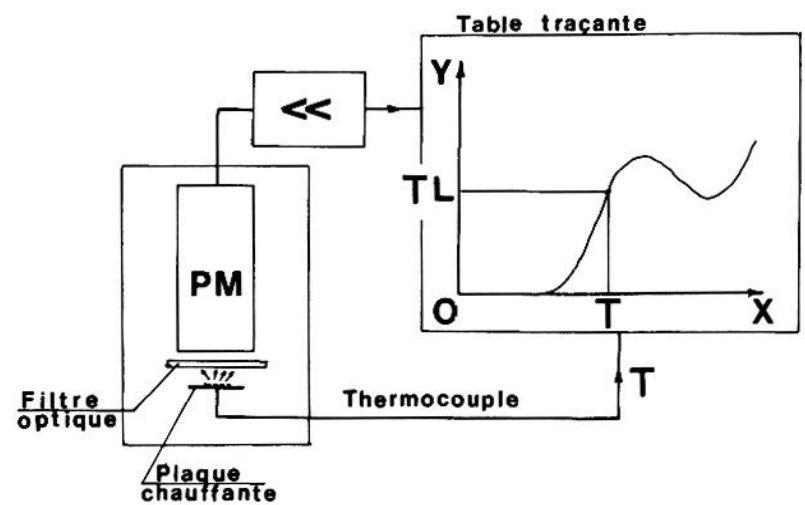

Fig. 1

l'oxygène de l'air qui pourrait engendrer une lumière parasite par la combustion de la matière organique incluse dans la roche, par exemple.

- la mesure de la lumière : elle est faite par un tube photo-multiplicateur (PM) qui délivre une tension à tout instant proportionnelle au flux lumineux émis par le minéral. Cette tension (TL) est mesurée sur une table traçante (XY) dont la plume décrit une courbe exprimant l'émission lumineuse de l'échantillon, selon l'axe $\mathrm{OY}$, en fonction de sa température $T$, donnée par un thermocouple, en OX.

Sur la figure 2 est représentée la thermoluminescence d'un échantillon de quartz (recuit à $700^{\circ}$ pour effacer l'excitation antérieure), irradié par les rayons $\beta$ d'une source de strontium $\left(\mathrm{Sr}^{90}\right)$. On voit qu'elle présente des maxima à $90^{\circ}, 195^{\circ}$ et $325^{\circ}$ (fig. 2, courbe 1). Audelà de $400^{\circ}$ l'émission lumineuse propre au support du minéral croît rapidement. Pour obtenir la thermo-

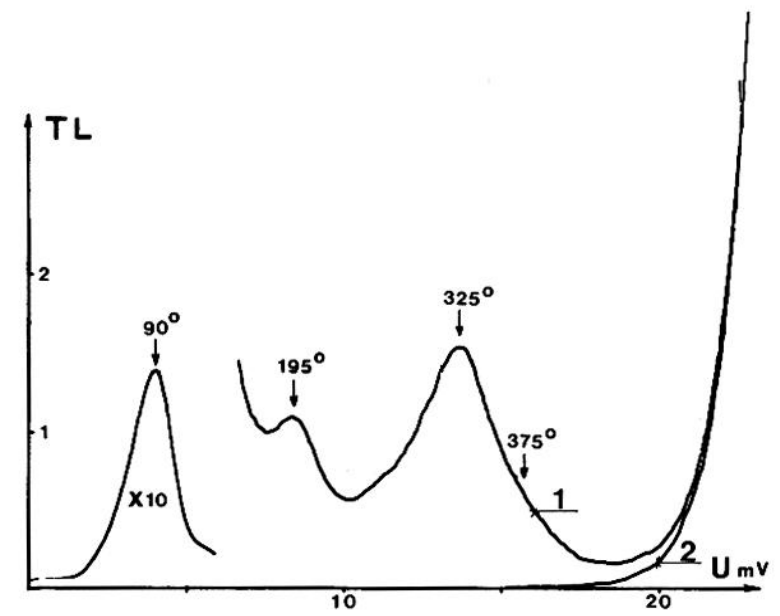

Fig. 2 - Courbe de thermoluminescence artificielle de $4 \mathrm{mg}$ de sable de Fontainebleau (granulométrie 125-160 $\mu \mathrm{m}$ ) recuit 7 heures à $700^{\circ} \mathrm{C}$ puis exposé a une dose de rayonnement $\beta$ de $2 \mathrm{krad}$. Vitesse de montée en température $10^{\circ} / \mathrm{sec}$. Filtre optique interposé entre le minéral et le PM : bleu (DH 380C' de MTO).

In abscisse : tension du thermocouple exprimé en $\mathrm{mV}(1 \mathrm{mV}$ correspond sensiblement à $25^{\circ}$ ).

lin ordonnée : la quantité de lumière mesurée par le photomultiplicateur en unités linéaires arbitraires. luminescence de l'échantillon il faut donc soustraire l'émission thermique du support. Pour cela on fait une deuxième chauffe au cours de laquelle seule l'émission du support se répète (courbe 2).

Seule la thermoluminescence obtenue à une température assez élevée $\left(\sim 300^{\circ} \mathrm{C}\right)$ représente bien la dose totale reçue par le minéral depuis la chauffe initiale. En effet, les pièges responsables de la thermoluminescence qui apparaît à des températures inférieure à $200-300^{\circ}$ se sont partiellement vidés de leurs électrons pendant la vie de la roche, l'agitation thermique se manifestant de façon non négligeable à la température ambiante. (Cette désexcitation spontanée accompagnée d'une faible émission lumineuse est connue sous le nom de phosphorescence). La lumière enregistrée à ces températures ne correspond alors qu'a une fraction de la dose reçue. Ce phénomène s'appelle le fading thermique. Il affecte d'autant plus les pics de thermoluminescence que leur temperature est plus basse. Ainsi sur la figure 2 qui montre la thermoluminescence mesuré dès l'irradiation faite, le pic à $90^{\circ} \mathrm{C}$ est présent. Il ne l'eut plus été 10 heures plus tard. La "vie moyenne" du pic (1) à $90^{\circ} \mathrm{C}$ est environ 3 heures à la température de $20^{\circ}$. A cette même température, les vies moyennes des pics à $195^{\circ} \mathrm{C}$ et à $360^{\circ} \mathrm{C}$ sont respectivement quelques années et plus de $10^{7}$ ans.

\section{B. Historique sommaire}

Un historique détaille serait long à faire. On peut consulter Thermoluminescence of Geological Materials [i] qui réunit des exposés théoriques et une diversité d'applications à la dosimétrie, la géophysique, et à la datation.

Les applications de la thermoluminescence commencent pendant les années 50 sur l'initiative de $F$. Daniel [2]. Une des plus importantes est la dosimétrie des rayonnements. C'est cet auteur qui, le premier, a envisagé de l'appliquer à la datation des céramiques et des roches, suivi par Houtermans [3] et Kennedy [4].

Une étape significative est le travail de M.S. Tite et J. Waine qui approfondissent le mécanisme de la thermoluminescence, ameliorent la technique de mesure, discutent la suppression des lumières parasites [5]. Une méthode de datation relative se développe alors de 1962 à 1966 [6] [7]. Nous nous y arrêtons car elle est interessante par sa simplicité et sa critique constitue une introduction aux problèmes de la datation absolue.

Il s'agit de relier la thermoluminescence d'une céramique à son âge. La méthode idéale consisterait à mesurer son accroissement en un temps donné, mais dans la plupart des cas, il faudrait que ce laps de temps soit très long pour que la thermoluminescence ait varié significa-

(1) Cettc expression est un raccourci pout : la vie moyenne des électrons sur les pièges responsables du pic à . . . 


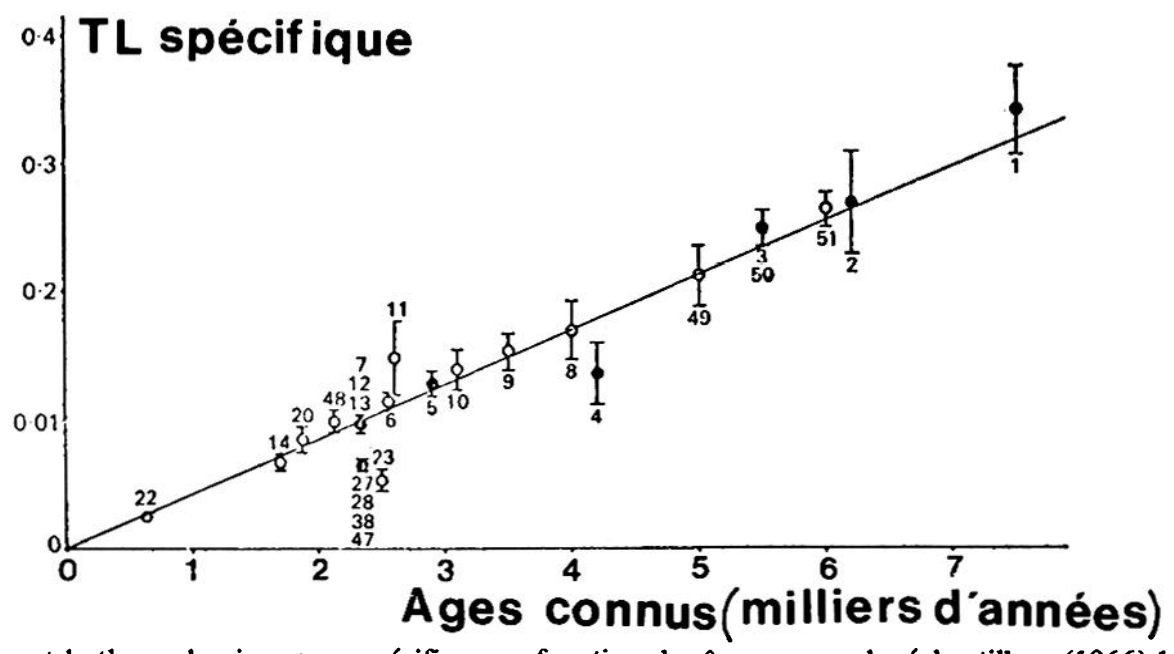

Fig. 3 - Courbe donnant la thermoluminescence spécifique en fonction des âges connus des échantillons $(1966) 1,2,3,4,5,9,10$ et 11 de Hasanlu, Iran ; 49, 50 et 51 de Susa ; Iran ; 8 du Balouchistan ; 6 et 7 de la plaine de Sybaris, Italie ; 20 de Le Muraglie, au dessus de la plaine de Sybaris, Italie ; 22, poterie Pecos des Etats-Unis sud ouest ; 23, 27, 28, 38, 47 et 48, poteries vernies noires de l'Agora d'Athènes. (d'après Ralph [15]).

tivement (2). On cherche donc à établir empiriquement le rapport TL/âge en travaillant sur un lot de céramiques d'âges connus. On ne peut s'attendre à un rapport TL/ âge unique puisque des céramiques faites d'argiles différentes ne contiennent pas en général la même proportion de radioéléments. D'autre part à doses reçues égales, elles ne produisent pas la même thermoluminescence. On cherche donc à caractériser chaque céramique par deux grandeurs :

- son niveau de radioactivité globale $\mathbf{R}$

- sa sensibilité au rayonnement $l_{\text {a }}$ qui sera représentée par sa réponse à une dose fixe, foumie par une source artificielle de référence (puisque la céramique elle même en tant que source a une intensité trop faible pour créer une thermoluminescence mesurable facilement en un temps court).

Soit $l_{n}$ la thermoluminescence naturelle de la céramique. On définit la thermoluminescence spécifique par le rapport :

$$
\mathrm{TL}_{\text {spec }}=\frac{\mathrm{l}_{\mathrm{n}}}{\mathrm{R} \mathrm{l}_{\mathrm{a}}}
$$

Cette thermoluminescence spécifique ainsi définie est indépendante de la nature minéralogique de la poterie. Elle est d'autant plus grande que la poterie est plus vieille. II reste à définir la "radioactivite" $R$. Dans le travail de $E$. Ralph et al., l'hypothèse est faite que ce sont les particules $\alpha$ des familles de l'uranium et du thorium qui jouent le principal rôle dans la gẻnèse de la thermoluminescence [8].

(2) Cela peut n'être pas vrai pour des minćraux tels que les zircons. Voir aussi à propos des feldspaths potassiques: Progress in Thermoluminescence dating in Edinburgh par H. Mc Kerrel et V. Mejdahl - Proceeding of the Nordic Conference on Thermoluminescence dating and other Archacometric Methods p 73. Uppsala University, Sweden 25-26 Novembre 1976.
On définit donc la grandeur $R$ comme le nombre de particules $\alpha$ sortant d'une surface donnée de la céramique, en $24 \mathrm{~h}$ par exemple. $\mathrm{R}$ est mesuré à l'aide d'un film de sulfure de Zinc où l'on a étalé la céramique réduite en poudre. Les particules $\alpha$ font dans le sulfure de Zinc des petits éclairs lumineux qui sont détectés par un photomultiplicáteur. Le rayonnement de référence appliqué à la céramique pour mesurer $1_{a}$ peut être un rayonnement $\alpha$ ou $\beta$.

La figure 3 montre d'après E. Ralf et al [8] les TL spécifiques d'un ensemble de céramiques en fonction de leurs âges connus (500 à 7000 ans). On constate que les points représentatifs se répartissent au voisinage d'une droite passant par l'origine. Cette droite permet d'obtenir l'âge d'une céramique d'après sa thermoluminescence spécifique avec une erreur de 20 à $25 \%$.

La précision limitée de cette méthode met en cause l'hypothèse faite du rôle prépondérant des $\alpha$ et oblige à considérer les autres rayonnements agissant dans la céramique. Pourtant, si l'on fait le bilan des doses libérées dans les céramiques d'après les concentrations moyennes connues des radioéléments, on constate que $80 \%$ environ de la dose qu'elles reçoivent vient des $\alpha$; mais on s'est aperçu que les $\alpha$ produisent beaucoup moins de thermoluminescence que les $\beta$, environ 10 fois moins a dose égale. Il s'ensuit que la contribution approchée des $\alpha$ à la dose totale n'est que $30 \%$. Ce n'est pas tout : les $\alpha$ sont des rayons de faible parcours $(\sim 20 \mu \mathrm{m})$ (ils sont engendrés par des impuretés radioactives localisées en général à la surface des cristaux de quartz et de feldspath. Ils pénètrent donc peu les gros minéraux de la céramique, quartz et feldspath décimillimétriques et n'y laissent qu'une faible énergie comparée à celle que les $\beta$ libérent dans tout le volume du minéral. De plus, si l'on ne s'attache qu'à la radioactivité dans la seule céramique, on ne tient pas compte du rayonnement $\gamma$ qui vient du sol environnant (cela résulte de la longue portée de ce rayonnement) (fig. 4). 


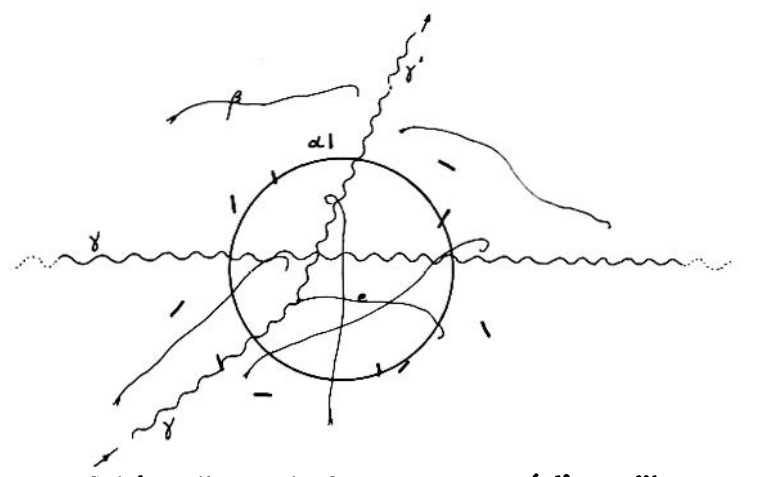

Fig. 4 - Schéma d'un grain de quartz entouré d'un milieu contenant des noyaux radioactifs émettant des particules $\alpha, \beta$ et $\gamma$. On a figuré arbitrairement des trajectoires de ces particules dans le plan de la figure. Chaque trajectoire est le chemin fait par une particule entre son point d'émission et celui où elle a cédé toute son énergie au milieu. Les trajectoires des $\alpha$ sont figurées par des traits droits noirs, celles des $\beta$ par des traits fins sinueux. Les $\gamma$ sont représentés par un trait ondulé. L'un d'eux a cédé une partie de son énergie à un électron à l'intérieur du grain de quartz (effet Compton).

Il était donc clair qu'il fallait développer une microdosimétrie appliquée aux céramiques et cela impliquait :

- les données sur la distribution des radioéléments dans les céramiques et le sol environnant,

- des études sur les actions spécifiques des rayonnements $\alpha, \beta$ et $\gamma$ sur les minéraux constituant les céramiques.

Les premières datations absolues faites par S.J. Fleming [9a] étaient un aboutissement naturel des travaux précédents.

Pour minimiser le rôle des $\alpha$ dans les calculs de dose, cet auteur utilisait les grains de quartz de l'ordre de $100 \mu \mathrm{m}$ qu'il pouvait extraire des céramiques. L'action de l'acide fluorhydrique, en éliminant une couche de l'ordre de $10 \mu \mathrm{m}$, achevait de rendre très petite la contribution des $\alpha, L a$ méthode des inclusions de Fleming consiste alors :

- à calculer la dose annuelle reçue par les grains de quartz de la part des $\beta$ de la céramique et des $\gamma d u$ sol. Or les $\gamma$ agissent par l'intermédiaire d'électrons rapides projetés, indifférenciables des rayons $\beta$. La dose annuelle totale $d$ n'a donc pour origine que des électrons rapides.

- à évaluer la dose intégrée depuis la chauffe initiale $D$. Pour cela, la thermoluminescence naturelle des quartz est comparé à la thermoluminescence provenant d'une dose connue d'un rayonnement artificiel. La source utilisee est une source $\beta\left(\mathrm{Sr}^{90}\right)$.

Le rayonnement d'une telle source simule bien le rayonnement que recevaient les quartz quand ils étaient dans la céramique.

- à calculer le rapport $\mathrm{D} / \mathrm{d}$ qui est l'âge absolu de la céramique. Dans cette détermination la thermoluminescence n'est qu'un agent de comparaison de doses de même origine physique.
Toutefois, le spectre en énergie des $\beta$ (ou électrons) agissant dans la céramique n'est pas identique au spectre en énergie des $\beta$ du strontium. Il ne peut donc y avoir équivalence absolue des actions de l'une ou l'autre dose : celle venant de la céramique et celle venant du strontium mais leurs effets sont pratiquement identiques.

Zimmerman développait sur la lancée la méthode des grains fins [ 10 a] laquelle exigeait un traitement détaillé de l'effet des $\alpha$.

L'ensemble des travaux poursuivis depuis 1968 à Oxford et dans d'autres laboratoires a permis d'affermir les techniques et d'approfondir maints problèmes. Pour un aperçu récent on lira avec profit l'article suivant : Thermoluminescent Dosimetry in Archaeological Dating par M.J. Aitken et S.J. Fleming [11].

Ce qui vient d'être dit concerne les céramiques. La datation des roches continue de poser des problèmes car une simple transposition des méthodes adaptées aux céramiques n'est pas immédiate. Les matériaux ne sont pas les mêmes. Les âges sont plus grands... Des exemples sont donnés dans le chapitre III. Le chapitre qui suit aborde la pratique de la datation par la thermoluminescence.

\section{II. - PRATIQUE DE LA THERMOLUMINESCENCE DES ROCHES}

\section{A. Introduction}

Les céramiques sont faites d'argile qui est une roche cryptocristalline où les radioéléments sont assez uniformément répartis et dans laquelle peuvent se trouver dispersés des grains plus gros $(100 \mu \mathrm{m})$ de quartz ou de feldspath. Les roches ou minéraux trouvés dans les foyers préhistoriques ou dans les coulées volcaniques, par exemple, sont de tailles quelconques et il n'y a plus de continuité dans la distribution de la radioactivité. Ils comprennent des minéraux peu fréquents dans les céramiques, silex, calcite. . qui exigent une approche particulière.

Nous serons donc amenés à étudier successivement :

- les roches et les radioéléments qu'elles contiennent : la mesure de leur concentration,

- la préparation des échantillons,

- la pratique de la thermoluminescence : détermination de la paléodose et le test du plateau,

- le calcul de la dose annuelle.

La méthodologie définie ci-dessous s'applique évidemment au cas plus simple des céramiques. 


\section{B. La roche}

1. Les portées approximatives des rayonnements $\alpha, \beta$ et $\gamma$ des radioéléments dilués dans la roche sont respectivement $20 \mu \mathrm{m}, 2 \mathrm{~mm}$ et $30 \mathrm{~cm}$ (densité du milieu 2.7).

Le tableau I donne des valeurs typiques des concentrations en uranium, thorium et potassium de quelques roches [12]:

TABLEAU I

\begin{tabular}{|l|c|c|c|}
\hline \multicolumn{1}{|c|}{ Roche } & $\mathrm{U}(\mathrm{ppm})$ & $\mathrm{Th}(\mathrm{ppm})$ & $\mathrm{K}(\%)(1)$ \\
\hline Argile & $2 \mathrm{à} 6$ & $8 \mathrm{à} 20$ & 2 à 8 \\
Granite & 3 & 12 & 4 \\
Basalte & 1.5 & 5 & 1.5 \\
Grès & 0,5 & 1.5 & $<1$ \\
Calcaire & $<0.1$ & $<0.3$ & $<0.1$ \\
Terre arable & 2 & 10 & 1 \\
\hline
\end{tabular}

(1) La concentration de lisotope $\mathrm{K}^{40}$ dans le potassium naturel est $0.0118 \%$

Le rapport de la concentration du thorium à celle de l'uranium est de l'ordre de 3 à 4.

Les radioéléments sont inégalement distribués dans toute roche mis à part l'argile. Le quartz n'en contient pratiquement pas. Une partie de l'uranium du granite est dans les joints des grains ou dans les zircons préférentiellement associés à la biotite...

\section{Mesure de la concentration en radioéléments}

La figure 5 représente les familles de $\mathrm{U}^{238}$ et $\mathrm{Th}^{232}$. La famille de $U^{235}$ n'est pas montrée car sa contribution en dose n'excède par $1 \%$.

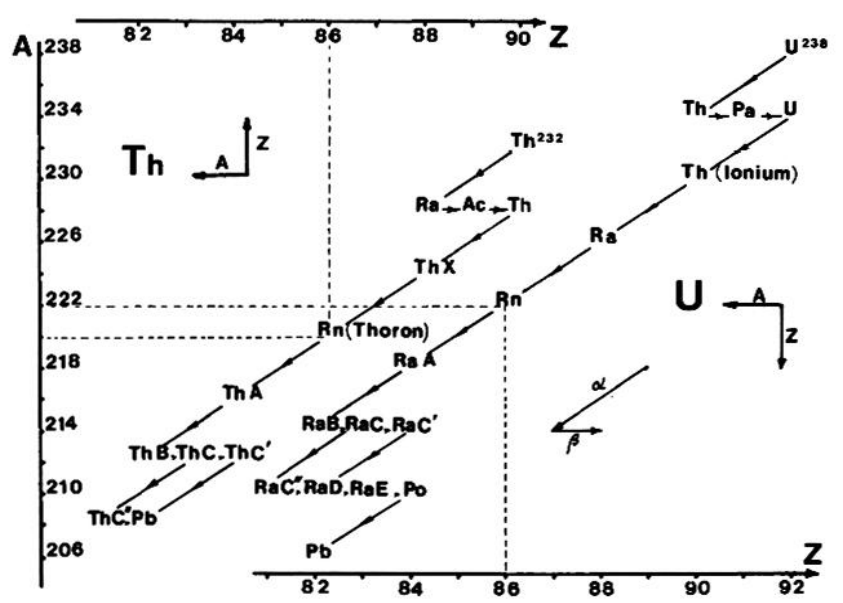

Fig. 5 - Les familles radioactives de $\mathrm{Th}^{232}$ et $\mathrm{U}^{238}$. Une flèche oblique exprime une transition $\alpha$ : le nombre de masse $A$ du noyau considéré décroît de 4 et le numéro atomique $Z$ de 2 unités ... Une flèche horizontale désigne une transition $\beta$.
Pratiquement, on mesure la concentration en uranium et thorium par l'une ou l'autre des 2 méthodes suivantes :

\section{Méthode des $\alpha$ (Oxford) [5]}

Il y a 8 (ou 6) $\alpha$ émis par les noyaux de la famille de l'uranium (ou du thorium) pour une désintégration du premier noyau.

Le détecteur est une couche mince de sulfure de zinc placée au-dessus d'un photomultiplicateur ou un semiconducteur de grande surface $\left(\sim 10 \mathrm{~cm}^{2}\right)$. On étale l'échantillon réduit en poudre sur la couche sensible et on totalise les $\alpha$ émis par les noyaux des deux familles. Cette méthode ne sépare pas leurs contributions respectives, mais on sait que le rapport $T h / U$ s'éloigne rarement beaucoup de 3.5. Si l'on met cette valeur dans le calcul on fait une erreur négligeable sur la dose $\beta$.

\section{Méthode des $\gamma[13]$}

Le noyau excité $\mathrm{RaC}^{*}$ formé par la désintégration $\beta$ de $\mathrm{RaC}$ émet $2 \gamma$ simultanés dont les énergies sont respectivement égales à 1.12 et $0.61 \mathrm{MeV}$ (fig. 6a). Leur mesure en coincidence par 2 compteurs séparés marque une désintégration de $\mathrm{RaC}$ de la famille de $\mathrm{U}^{238}$. ThC", de la famille de $\operatorname{Th}^{232}$, est caractérisé par une cascade similaire de $2 \gamma(0.58$ et $2.61 \mathrm{MeV})$ : fig. $6 \mathrm{~b}$.

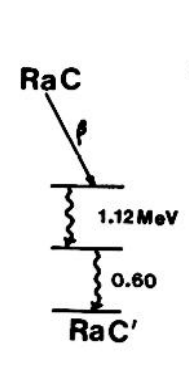

a

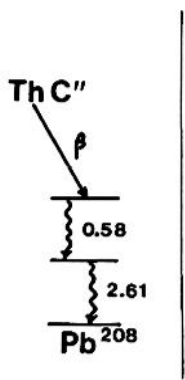

b

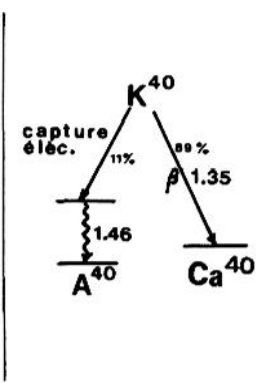

C
Fig. 6 - a - La partie du schéma de désintégration de RaC concernant la cascade de $2 \gamma$ utilisée pour mesurer la concentration en uranium dans un échantillon de roche.

b - La partie du schéma de désintégration de ThC" concernant la cascade de $2 \gamma$ utilisée pour mesurer la concentration en thorium.

c - Le schéma de désintégration de $\mathrm{K}^{\mathbf{4 0}}$ (abondance dans le potassium naturel : $0,0118 \%$ ).

De la fréquence des cascades enregistrées associées à $\mathrm{RaC}$ ou ThC", on remonte aux concentrations respectives en uranium et en thorium.

L'une ou l'autre de ces deux méthodes donne un re. sultat incorrect si l'unique élément gazeux de la filiation de l'uranium ou du thorium, le radon $\left(\operatorname{Rn}^{222}\right)$ ou le thoron $\left(\mathrm{Rn}^{220}\right)$, s'échappe de la roche. Si c'est le cas, les noyaux de radon concernés ne sont pas comptés ni leurs descendants. Les concentrations trouvées sont trop faibles.

Dans le cas de la méthode des $\boldsymbol{\gamma}$ on place l'échantillon de roche $(\sim 20 \mathrm{~g})$ dans une boîte étanche et on attend 
que le radon soit en équilibre radioactif avec le radium, soit un mois.

Dans le cas de la méthode des $\alpha$, on ne peut procéder de la même manière puisque les $\alpha$ doivent venir de la couche superficielle en contact avec le détecteur, alors que le radon tend à remplir la boîte. . . La méthode appropriée à ce cas est décrite par Aitken et Fleming.

Le départ du radon est certainement plus important dans la première méthode puisque les $\alpha$ viennent de la couche superficielle du minéral. Dans le cas de la seconde, il est en général négligeable car on utilise une vingtaine de grammes de roche compacte.

Pour mesurer le potassium dont le schéma de désintégration est montré par la figure $6 c$, on peut détecter ses $\gamma$ on utiliser la spectrométrie d'absorption.

\section{Préparation des échantillons}

Les minéraux les plus utiles en datation sont actuellement :

- le quartz : galets centimétriques, quartz du granite, sable, grès, quartzite, ques),

- les feldspath des granites (ou des arènes graniti-

- les silicates microcristallins des argiles cuites,

- le zircon, le silex, la calcite.

Les plagioclases des roches volcaniques n'ont pratiquement pas été utilisés jusqu'à présent à cause de leur fading [14]. Cependant on verra plus loin des résultats encourageants (chapitre III).

La préparation des échantillons emprunte à la minéralogie ses techniques : broyage, lavages, tamisage, séparation magnétique. . [15].

Chaque minéral exige un traitement qui dépend de sa forme initiale et de l'état de division souhaité. Ainsi un minéral finement divisé $(\sim 10 \mu \mathrm{m})$ est déposé par sédimentation sur des disques : c'est la technique des grains fins de Zimmerman [10 b].

La taille la plus convenable des grains de quartz est voisine de $100 \mu \mathrm{m}$. On peut les séparer magnétiquement après tamisage s'ils sont dans une argile cuite.

Les phénocristaux millimétriques doivent être broyés au mortier. Ce traitement doit être conduit avec précaution car il peut engendrer une thermotriboluminescence.

\section{Détermination de la paléodose}

Le minéral est partagé en échantillons de masses égales sur des supports, coupelles ou disques. La thermoluminescence naturelle (TLN) est obtenue sur un premier échantillon (courbe 0 de la figure 7a). Puis on

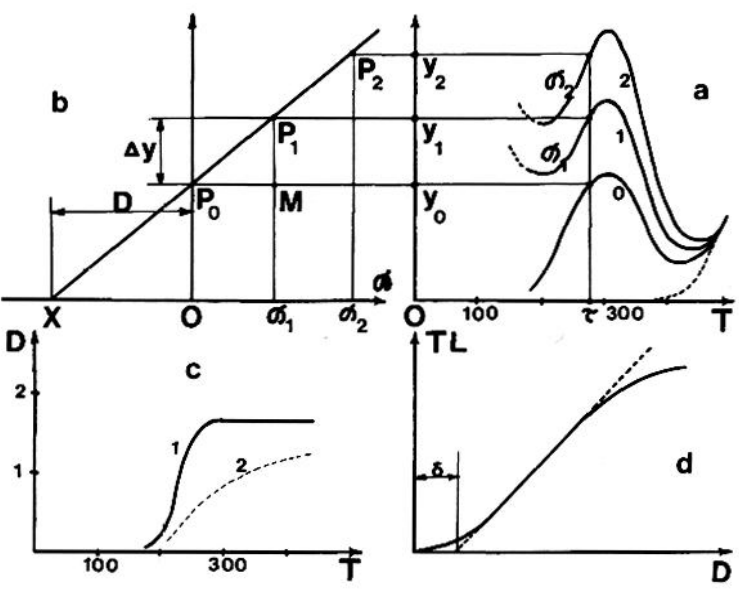

Fig. 7 - a - Représentation schématique de 3 courbes de TL obtenues dans les cas suivants :

Courbe $0:$ TLN d'un échantillon archéologique, correspondant en principe à la paléodose qu'il a reçue

Courbe 1: TL d'un échantillon identique au premier mais qui a reçu une dose artificielle supplémentaire $\mathcal{O}$

Courbe 2: même chose pour une dose $\mathscr{O}_{2}^{1}>\mathscr{O}_{1}$

b - Graphique représentant les lumières émises à la temperature $\tau$ par les échantillons précédents en fonction de la dose qu'ils ont reçue. L'abscisse $X$ de l'intersection de la droite menée par $P_{0}, P_{1}, P_{2}$ correspond à la dose reçue dans le passé, la paléodose D. Par les triangles semblables $X O P_{0}$ et $P_{0} M P_{1}$ on $a$ :

d'où :

$$
\frac{O X}{P_{0} M}=\frac{D}{\Phi_{1}}=\frac{O P_{0}}{M P_{1}}=\frac{y_{0}}{\Delta y}
$$

$$
D=\frac{y_{0}}{\Delta y} \mathcal{D}_{1}
$$

c - Le test du plateau : D est porté en fonction de la température $T=\tau$ où les lumières ont été mesurées. La courbe 1 présente un plateau au-delà de $300^{\circ}$.

La courbe 2 représente le cas d'un fading anomal.

d - Profil réel de la croissance de la TL en fonction de la dose. Très souvent on a un comportement supralinéaire aux faibles doses qui oblige d'ajouter la dose $\delta$ à la paléodose trouvée par extrapolation. Aux grandes doses la courbe s'incurbe et tend vers une valeur stationnaire.

mesure la thermoluminescence d'échantillons exposés à des doses artificielles $\boldsymbol{O}_{1}, \boldsymbol{O}_{2} \ldots$ qui s'ajoutent à la dose antérieurement reçue $\mathrm{D}$ (paléodose). On obtient les courbes $1,2 \ldots$ : ce sont les réponses du minéral aux doses $\mathrm{D}+\boldsymbol{O}_{1}, \mathrm{D}+\boldsymbol{O}_{2} \ldots$ Le graphique de la figure $7 \mathrm{~b}$ représente la thermoluminescence des différents échantillons en fonction de la dose reçue. Si la quantité de lumière varie linéairement avec la dose, il est alors possible de déterminer la paléodose $D$ par extrapolation linéaire.

Pour que la valeur de $\mathrm{D}$ ainsi trouvée ait un sens il faut qu'elle soit indépendante de la température $r$ à laquelle on a mesuré la thermoluminescence. Le test $d u$ plateau [16] consiste à le vérifier en portant $\mathrm{D}$ en fonction de $\tau$ sur un graphique tel que celui de la figure $7 \mathrm{c}$. 
Dans les bons cas $\mathrm{D}$ devient constant au-delà de $300^{\circ}$ environ (courbe 1 ).

D'un point de vue physique le test du plateau revient à contrôler que la TLN a bien pour origine l'action d'un rayonnement : la thermoluminescence artificielle (TLA) a alors le même profil en fonction de la température que la TLN.

Si D n'est pas constant cela peut signaler la présence d'une lumière parasite ou un fading anomal de la TLN.

La lumière parasite peut provenir d'une chimioluminescence ou d'une thermo-triboluminescence (libération au cours de la montée en température des contraintes provenant de l'écrasement du minéral). Elle est fortement réduite ou éliminée si la chauffe est faite en atmosphère neutre.

Le fading anomal est distinct du fading thermique décrit au chapitre I. Nous avons vu que le fading thermique est dù à l'agitation thermique à la température ambiante qui vide progressivement de leurs électrons les pièges responsables de la thermoluminescence "basse température" ( $\left.\mathrm{T}<250^{\circ}\right)$. Elle affecte de façon négligeable les pièges associés à la thermoluminescence qui se développe au-delà de $300^{\circ}$ environ. Toutefois, il arrive que la TL qui se développe au-delà de cette température puisse disparaître partiellement en un temps court ( $\sim$ semaine) malgré une conservation du minéral à la température de l'azote liquide. Ce phénomène s'appelle fading anomal [14].

En général, le fading anomal diminue vers les hautes températures et la valeur de $\mathrm{D}$ trouvée croît alors avec la température comme le montre la courbe 2 de la figure 7c. C'est pourquoi on cherche à étendre la mesure de la thermoluminescence aux plus hautes températures possibles. C'est l'émission thermique du support, très vite prépondérante, qui fixe la limite $\left(\mathrm{T}=500-550^{\circ}\right)$.

En conclusion, le test du plateau informe sur la possibilité d'obtenir un âge ; toutefois il arrive qu'il n'y ait pas de plateau sans que cela soit dû à l'émission d'une lumière parasite ou au fading anomal. Un traitement approprié du minéral permet parfois d'effectuer la datation [23].

Remarquons enfin que l'hypothèse d'une thermoluminescence proportionnelle à la dose n'est correcte en général ni aux faibles doses ni aux grandes doses:

- aux faibles doses on observe souvent une variation du type parabolique ; ce comportement est dit supralinéaire.

- aux fortes doses la thermoluminescence devient stationnaire ; on dit qu'il y a saturation.

- aux valeurs intermédiaires, la courbe de variation peut être approchée par une droite qui coupe l'axe des $X$ en un point d'abcisse $\delta, \delta$ exprime l'effet de la supralinéarité. Des valeurs de quelques centaines de rad sont courantes pour le quartz et le feldspath.

\section{La dose annuelle}

Pour évaluer la dose reçue annuellement par le minéral on détermine séparément la dose interne $\mathrm{d}_{\mathrm{i}}$ et la dose externe $d_{e} \cdot d_{i}$ représente l'action des rayons de faible parcours $\alpha(20 \mu \mathrm{m}), \beta(2 \mathrm{~mm})$ qui ne peuvent donc provenir que du voisinage immédiat du minéral.

$\mathrm{d}_{\mathrm{e}}$ représente l'action des rayons $\gamma$ et cosmiques.

$\mathrm{Vu}$ la portée des $\gamma$, c'est l'ensemble de la roche et du sol dans une sphère de $30 \mathrm{~cm}$ de rayon centrée sur le prélèvement qui est en jeu. La dose cosmique dépend de la latitude, de l'altitude et de la profondeur dans le sol.

$\mathrm{La}$ dose totale reçue annuellement est alors la somme des deux termes précédents : $d=d_{i}+d_{e}$

La contribution à $d_{e}$ des gamma émis par les radioéléments du sol peut être calculée de la même manière que va l'être $d_{i}$ à partir des concentrations mesurées des radioéléments dans le sol entourant la roche (voir l'application numérique à la fin du paragraphe). Quant à la dose cosmique, elle est en France à basse altitude 25 à $30 \mathrm{mrad} / \mathrm{an}$. Elle tombe à une valeur de 13 à $14 \mathrm{mrad} / \mathrm{an}$ à $1 \mathrm{~m}$ de profondeur dans le sol [17 a] et décroit lentement à des profondeurs plus grandes. On peut mesurer globalement $d_{e}$ (gammas du sol + cosmique) en exposant des dosimètres thermoluminescents très sensibles $\left(\mathrm{SO}_{4} \mathrm{Ca}(\mathrm{Dy})\right)$ au point considéré du sol pendant des temps allant de 1 mois à 1 an [17 b] [18].

Dans le cas idéal où les radioéléments peuvent être supposés uniformément répartis dans la roche, comme cela arrive dans les céramiques à grains fins, le débit de dose d'un rayonnement donné (par exemple le rayonnement $\beta$ ) émis par un ensemble de noyaux (par exemple les noyaux de la famille de l'uranium) s'écrit :

$$
d_{i}(\beta)\left(\text { uranium) }=\sum \eta_{k} \epsilon_{k}\right.
$$

où $\eta_{k}$ désigne le nombre de désintégrations par an et par gramme de la roche du radioélément $k$ et $\epsilon_{K}$ l'énergie moyenne de son rayonnement. Le bilan des doses annuelles, utilisant les données les plus récentes, donné par Bell [19], figure dans le tableau II.

TABLEAU II

\begin{tabular}{|c|c|c|c|}
\hline & \multicolumn{3}{|c|}{ doses (mrad/an) } \\
\hline & \multicolumn{3}{|c|}{ nature du rayonnement } \\
\hline & $\alpha$ & $\beta$ & $\gamma$ \\
\hline $\begin{array}{l}1 \mathrm{ppm} \mathrm{d} \mathrm{U}^{238} \\
1 \mathrm{ppm} \text { de } \mathrm{Th}^{232}\end{array}$ & $\begin{array}{r}278.3 \\
73.8\end{array}$ & $\begin{array}{r}14.62 \\
2.86\end{array}$ & $\begin{array}{r}11.48 \\
5.14\end{array}$ \\
\hline $1 \% \mathrm{~K}_{2} \mathrm{O}$ & & 68.2 & 20.5 \\
\hline
\end{tabular}

Doses annuelles spécifiques. Les doses relatives à l'uranium et au thorium totalisent les énergies respectives des rayonnements $\alpha \beta$ et $\gamma$ émis par les radioéléments de la filiation de $\mathrm{U}^{238}$ et $\mathrm{Th}^{232}$, montrées par la figure 5, à l'équilibre radioactif et sans échappement du radon. 
Pour obtenir la dose annuelle apportée par les rayons $\beta\left(d_{i}(\beta)\right)$ il suffit d'additionner les produits des chiffres de la $2^{\text {eme }}$ colonne du tableau II par les concentrations respectives en uranium, thorium et potassium dans la roche. Celle qui correspond aux rayons $\alpha$ se calcule de la même manière mais rappelons que l'efficacité des $\alpha$,pour produire la thermoluminescence, est sensiblement plus petite (à dose égale) que celle des $\beta$. Or les sources artificielles de référence utilisées étant des sources $\beta$ on doit exprimer la dose $\alpha$ sous forme d'une dose $\beta$ qui produirait la même TL : cela revient pratiquement à multiplier la dose $\alpha$ par un certain facteur k. La dose interne totale annuelle est alors:

$$
d_{\mathbf{i}}=\mathbf{d}_{\mathbf{i}}(\beta)+\mathbf{k d}_{\mathbf{i}}(\alpha)
$$

On obtient le facteur $k$ en comparant les TL produites respectivement par l'exposition des échantillons à des sources $\alpha$ et $\beta$ connues. Un ordre de grandeur moyen de $k$ est 0.1 . C'est la valeur qui est prise dans l'application numérique numérique suivante.

\section{Application numérique}

Cas d'une céramique enfouie à $1 \mathrm{~m}$ de la surface du sol :

$$
\begin{aligned}
& \text { concentration en uranium : } 2 \mathrm{ppm} \\
& \text { " "thorium : } 8 \mathrm{ppm} \\
& \text { " } \quad \mathrm{K}^{2} \mathrm{O}: 3 \%
\end{aligned}
$$

Doses annuelles :

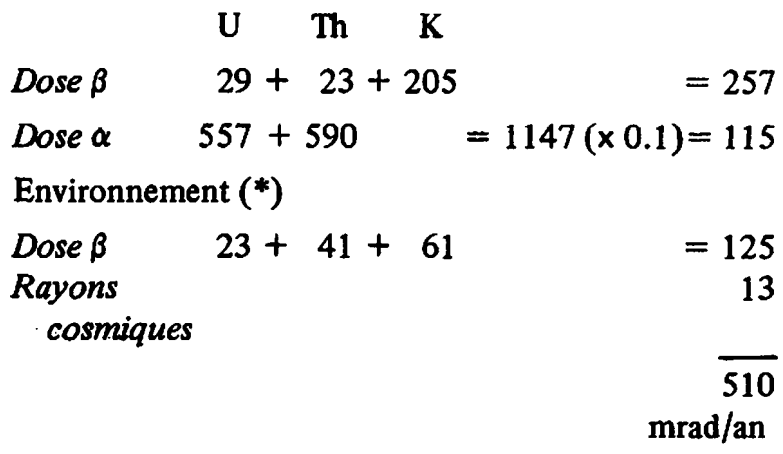

Si la phase minérale extraite de la roche a une granulométrie excédant par exemple $50 \mu \mathrm{m}$, la dose qui lui est apportée par les rayons $\alpha$ ne peut plus être considérée comme uniforme puisqu'ils ne peuvent aller à plus de $20 \mu \mathrm{m}$ en moyenne vers l'intérieur d'un grain ; la dose moyenne qu'il reçoit est alors inférieure à la dose règnant à l'extérieur du grain.

La même remarque s'applique à l'irradiation par les rayons $\beta$ si le diamètre du grain excède le parcours de ces rayons. A titre d'exemple, un grain de quartz supposé sphérique de $5 \mathrm{~mm}$ de diamètre plongé dans une phase contenant une distribution uniforme de potassium $\left(\epsilon_{\max }=1.35 \mathrm{MeV}\right)$ reçoit une dose moyenne égale à $17 \%$ de ce que recevrait un grain de $100 \mu \mathrm{m}$ [20].

A l'inverse un radioélément peut se trouver plus concentré dans un minéral que dans l'environnement

${ }^{*}$ ) On suppose ici que les concentrations en radioéléments dans le sol entourant la céramique y sont les mêmes que dans cette dernière. de celui-ci d'où une dose interne plus grandequ'alentour. Ainsi un feldspath à $10 \%$ de $\mathrm{K}^{2} \mathrm{O}$ a en son centre une dose propre de $682 \mathrm{mrad} / \mathrm{an}$ s'il a plus de $3 \mathrm{~mm}$ de diamètre.

Enfin, l'évaluation de $\mathrm{d}_{\mathbf{i}}$ exige de connaître la concentration en eau dans la roche si elle est poreuse. C'est le cas des argiles cuites. Pratiquement si l'on appelle $\mathrm{d}^{\mathrm{s}}{ }_{\mathrm{i}}$ la dose relative au prélèvement $\mathrm{sec}$, la dose vraie est égale à :

$$
\mathbf{d}_{\mathbf{i}}=\theta \mathrm{d}_{\mathbf{i}}^{\mathbf{s}} \quad \text { où } \quad \theta
$$

a pour expression :

$$
\theta=\frac{\text { poids du prélèvement sec }}{\text { poids réel moyen du prélèvement en place }}
$$

Il est donc important de connaître l'hydrologie du site. On trouvera un exemple dans la référence [21].

\section{III. - APPLICATIONS}

Les principes de la datation des roches brâlées et des céramiques ont été exposés ci-dessus. Ce demier chapitre passe en revue quelques applications récentes, arbitrairement choisies.

Les méthodes applicables aux céramiques développées à Oxford sont probantes et l'erreur peut descendre à $5 \%$ dans les bons cas si l'on parvient à bien définir la concentration moyenne en eau dans et autour de la céramique. Les applications aux roches sont moins développées : le domaine d'âge est plus étendu et on trouve dans les roches des minéraux différents de ceux qui composent les céramiques ou dans des situations différentes.

Quelques problèmes ont été exposés par M.J. Aitken au Symposium d'Edimbourg [22]. L'un de ceux qui se rencontrent fréquemment dans l'exploitation des quartz, par exemple, est l'absence d'un plateau sur la courbe qui exprime en fonction de la température le rapport de la thermoluminescence naturelle à la thermoluminescence artificielle (cf § II C). Nous avons proposé une méthode pour obtenir une valeur non ambiguë de la paléodose dans ce cas [23].

Dès 1972, A.G. Wintle a daté un fragment de cristal de roche chauffé trouvé près de restes humains dans l'ile de Ceylan [24].

Une application archéologique récente est la datation du site de Pincevent par Poupeau et al. [25]. Ces auteurs utilisent un morceau de granite issu d'un foyer de ce site. Pour s'affranchir du problème posé par l'eau, ils déterminent séparément la dose $\gamma$ de l'environnement du granite en exploitant un grès du même foyer qui n'a reçu que cette dose, ne contenant pratiquement pas de radioéléments. La dose reçue par les grains de quartz du granite, de diamètre moyen voisin de $0,5 \mathrm{~mm}$, comprend la dose $\gamma$ déjà connue 
et la dose déposée par les $\beta$ du potassium des feldspath et des éléments lourds (uranium, thorium) situés dans les joints des grains et sous forme d'inclusions dans les quartz eux-mêmes. La dose moyenne reçue par les quartz a été déterminée par le calcul. L'âge trouvé $13900 \pm 2000$ est en bon accord avec l'âge C.14 de $11000 \pm 1000$.

L'exploitation des silex chauffés est gênée par une lumière parasite provenant de leur écrasement. C'est pourquoi Goksu et al. préconisent de travailler sur des lames du minéral (épaisseur 0,3 à $0,4 \mathrm{~mm}$ ), plutôt que sur des grains [26]. Aitken et Wintle ont publié la datation du site de Terra Amata, obtenue selon cette technique [27]. L'article contient une étude détaillée des implications de la variation de la dose $\beta$ artificielle en fonction de la profondeur dans la lame et de l'autoabsorption de la lumière émise. L'âge trouvé est 230.000 \pm 40.000 ans.

La datation des foyers préhistoriques de la région parisienne utilisant des silex et des grès est en cours au C.F.R. Il a été montré que le découpage des silex en lames n'est pas toujours nécessaire [28].

L'inversion magnétique de Laschamp a motivé plusieurs tentatives de datation des coulées volcaniques d'aimantation inverse connues de la Chaîne des Puys [29]. Des mesures faites au CFR sur le quartz d'une enclave granitique trouvée dans la lave de Laschamp fournit un âge de $35.000 \pm 3000$ ans [30]. Huxtable et Aitken donnent $25.800 \pm 2200$ ans pour la coulée de Royat à partir d'un sédiment situé sous la lave [31].

La coulée de lave d'Olby a été datée de deux manières : l'une est fondée sur des galets de quartz situé à $5-10 \mathrm{~cm}$ sous la lave, l'autre sur la lave elle-même. L'âge trouvé avec les galets est $38000 \pm 6000$ ans [32]. La datation de la lave utilise la thermoluminescence à haute température des plagioclases (500 $700^{\circ} \mathrm{C}$ ) et met en jeu des pièges non affectés par le fading anomal [33]. L'âge trouvé pour la lave d'Olby est $44100 \pm 6500$ ans en bon accord avec l'âge précédent.

A.G. Wintle a discuté les effets de la méthode de préparation de la calcite sur sa thermoluminescence [34].

Pour un aperçu des derniers développements méthodologiques, renvoyons aux comptes rendus du $1^{\text {er }}$ séminaire des spécialistes de la datation par la thermoluminescence tenu à Oxford en juillet 1978 .

Manuscrit reçu en septembre 1977

\section{BIBLIOGRAPHIE}

[1] McDougall D.J., 1968. - Thermoluminescence of Geological Materials, Academic Press.

[2] Daniels F., Boyd C.A. and Saunder D.F., 1953. Thermoluminescence as a research tool. Science, $117,343$.
[3] Houtermans F.G., Jager E., Shon M., Stauffer H, 1957. - Messungen der thermolumineszenz Als Mittel zur Untersuchung der termischen undder Strhlumgsgeschichte von naturlichen Mineralien und Gresteinen. Ann. d. Physik, 20, 283.

[4] Kennedy G., Knopff L., 1960. - Dating by thermoluminescence. Archeology, 13, 147.

[5] Tite M.S., Vaine J., 1962. - Thermoluminescent dating : a re-appraisal. Archaeometry, 5, 53.

[6] Aitken M.J., Tite M.S., Reid J., 1962. - Thermoluminescent dating of ancient ceramics. Nature, $202,1032$.

[7] Ralph E.K., Han M.C., 1966. - Dating of pottery by thermoluminescence. Nature, 210, 245.

[8] Ralph E.K., Han M.C., 1969. - Potential of thermoluminescence in supplementing radiocarbon dating. World-Archaeology, 1.

[9 a] Flemming S.J., 1966. - Study of thermoluminescence of crystalline extracts from pottery. Archaeometry, 9, 170.

[9 b] 1970. - Thermoluminescent dating : refinement of the quartz inclusion method, Archaeometry, $12,133$.

[10 a] Zimmermann D.W., 1967. - Thermoluminescent dating using fine grains from pottery. Archaeometry, 10, 26.

[10 b].1971. - Archaeometry, 13, 29.

[11] Attix F.H., 1972. - Topics in Radiation Dosimetry, Academic Press.

[12] 1974. - Handbook of geochemistry, Springer Verlag, Berlin.

[13] Reyss J.L., Yokoyama Y., Duplessy J.-C., 1978 A rapid determination of sedimentation rates by non destructive $\boldsymbol{\gamma}-\boldsymbol{\gamma}$ coincidence spectroscopy, Deep Sea Res., 25, 491.

[14] Wintle A.G., 1973. - Anomalous fading of thermoluminescence in mineral samples. Nature, 245, 143.

[15] Parfenoff A., Pomerol C., Tourenq J., 1970. Les minéraux en grains, Masson.

[16] Aitken M.J., 1968. - Thermoluminescent dating in archaeology : introductory review, In : Thermoluminescence of Geological Materials, (McDougall D.J. Ed.) 369.

[17 a] Aitken M.J., 1968. - Low-level environmental radiation measurements using natural calcium fluoride, Proc. 2nd Int. Conf. Luminescence Dosimetry, Gatlinburg, 1968 (Conf. 680920, NBS, Springfield, Virginia).

[17b]Mejdahl V., 1970. - Measurement of environInental radiation at archaeological excavation sites. Archaeometry, 12, 147. 
[18] De tels dosimètres sont préparés au Centre d'Etudes Nucléaires de Fontenay-aux-Roses (Section Technique d'Etudes de Protection, Institut de Protection et do Sûreté Nucléaires).

[19] Bell W.T., 1977. - Thermoluminescence dating : revised dose-rate data. Archaeometry, 19, 99.

[20] Valladas G. - Exploitation de la thermoluminescence du quartz, en préparation.

[21] Valladas H., 1976. - Tentative de datation d'un four médiéval, Mémoire de maîtrise d'archéologie, présenté à l'Université de Paris $I$.

[22] Aitken M.J., 1966. - The thermoluminescent dating : some problems, Edinburgh Symposium, March 1966.

[23] Valladas G., 1978. - A problem encountered in dating quartz by thermoluminescence, Proceedings of the 18th Intern. Symposium on Archaeometry and Archaeological Protection, Bonn (Germany) March 1978.

[24] Wintle A.G. and Oakley K.P., 1972. - Thermoluminescent dating of fired rock-crystal from Bellan Bandi Palassa, Ceylon. Archaeometry, 14, 277.

[25] Poupeau G., Sutton S.R., Walker R.M. and Zimmerman D.W., 1976. - Thermoluminescent dating of fired rocks : application to the site of Pincevent, France, Proceedings IXe Congrès de l'Union Internationale des Sciences Préhistoriques et Protohistoriques, Nice, Septembre 1976.

[26] Göksu H.Y. and Fremlin J.H., 1972. - Thermoluminescence from unirradiated flints : regeneration thermoluminescene. Archaeometry, 14, 127.
[27] Wintle A.G. and Aitken M.J., 1977. - Thermoluminescence dating of bumt flint : application to a lower palaeolithic site, Terra Amata. Archaeometry, 19, 111.

[28] Valladas H., 1978. - Thermoluminescence dating of burnt stones from prehistoric site,. A specialist seminar on thermoluminescence dating, Oxford, July 1978 (Vol. 3 of the Council of Europe's PACT Journal.)

[29] Gillot P.Y., Labeyrie J., Laj C., Valladas G., Guérin G., Poupeau G. and Delibrias G., 1978. - Age of the Laschamp paleomagnetic excursion revisited, Submitted. Earth and Planet Sc. Lett., 42, 444.

[30] Gillot P.Y., Valladas G. and Reyss J-L., 1978. Dating of lava flow using a granitic enclave : application to the Laschamp magnetic event, A specialist seminar on thermoluminescence dating, Oxford, July 1978 (Vol. 3 of the Council of Europe's PACT Journal).

[31] Huxtable J., Aitken M.J. and Bonhommet N., 1978. - Thermoluminescence dating of sediment baked by lava flows of the Chaine des Puys, $\mathrm{Na}$ ture, 275, 207.

[32] Valladas G. and Gillot P.Y., 1978. - Dating of the Olby lava flow using heating quartz pebbles : some problems, A specialist seminar on thermoluminescence dating, Oxford, July 1978 (Vol. 3 of the Council of Europe's PACT Journal).

[33] Valladas G., P.Y. Gillot and Guerin G., 1978. Dating plagioclase ? Idem.

[34] Wintle A.G., 1975. - Effects of sample preparation on the thermoluminescence characteristics of calcite. Modern Geology, 5, 165. 